\title{
Experimental Investigation of Feature Quantity in Sound Signal and Feeling Impression Using PCA
}

\author{
Yusuke Kawakami, Tetsuo Hattori* \\ Graduate School of Engineering, Kagawa University, 2217-20, Hayashi-Cho \\ Takamatsu, Kagawa 761-0396, Japan \\ E-mail: hattori@eng.kagawa-u.ac.jp \\ Hiromichi Kawano \\ NTT Advanced Technology Corporation, 19-18, Nakamachi \\ Musashino, Tokyo 180-0006, Japan \\ E-mail: hiromichi.kawano@ntt-at.co.jp \\ Tetsuya Izumi \\ Micro-Technica Co., Itd., 3-12-2, Higashi-Ikebukuro \\ Toshima, Tokyo 170-0013, Japan \\ E-mail: t-izumi@microtechnica.co.jp
}

\begin{abstract}
This paper describes experimental investigation of the relationship between feature quantity of sound signal and feeling impression using PCA (Principal Component Analysis). As the feature quantity, we use Fluctuation value and sum of squared errors (Residual) which is calculated by regression analysis of sound signal, in the same way as our previous paper. In order to investigate the feeling impression and effect from sound signal, we use a questionnaire survey method, that is, we ask some examinees to evaluate their feeling impression about sound (music) that we provide. As a result, we have found that the feeling response of examinees can be classified into three groups by a clustering analysis. And we have verified the feeling impression effects depending on each group of examinees and four kinds of frequency zone of sound signal from the results of PCA. In this paper, we also discuss the analysis results on the Kansei (or feeling) effect.
\end{abstract}

Keywords: Signal processing, Fluctuation, Intercept, Sum of squared errors, Feeling impression, PCA

\section{Introduction}

Recently, $1 / f$ fluctuation in various fields of signal has been actively researched, and it brings about an effect of such healing as a human being psychologically feels at ease, if there is a $1 / f$ relation between the power spectrum of the signal and the frequency $f^{1-7}$. However, we focused that the power spectrum have same fluctuation but the distribution are different. And we doubted the strong influence of the emotional impression factors other than fluctuation value. ${ }^{8}$

* Graduate School of Engineering, Kagawa University, 2217-20, Hayashi-Cho, Takamatsu, Kagawa 761-0396, Japan

E-mail: hattori@eng.kagawa-u.ac.jp 
Therefore, in the previous research, we have defined three kinds of parameters such as fluctuation value (or Fluctuation), intercept (or Intercept), and sum of squared errors (or Residual) as feature quantity in sound signal obtained from the calculation of the signals' fluctuation degree. And we have investigated the relation between feeling impression and those parameters, by using multiple regression analysis ${ }^{8}$. And we eliminate "Intercept" from the analysis, because this quantity (or parameter) is substantially equal to the volume of sound ${ }^{8}$.

Moreover we had considered possibility of the effect of feeling impression from frequency domains. So we divided into three frequency domains (Low Frequency (LF); 0 300Hz, Middle Frequency (MF); 300 1000Hz, High Frequency (HF); 1000 22050Hz) and analyzed each domain ${ }^{10,11,16}$.

As the results, we have understood that feeling impression have an impact on Residual more than Fluctuation, especially high frequency. In the regression analysis, we can be seen the impact of the sound features for the evaluation value of individual sensibility adjective items (for example, lightness, quickness, etc.). Although, it is difficult to capture the main factors on the relation between feature quantity of sound which presented to examinees, and feeling impression of examinees.

In this paper we analyze the affect of the feeling impression, from the music's Fluctuation and Residual, by use of principal component analysis. At first, we investigate the feeling impression of the music by using questionnaire survey. Then, from result we separate the examinees into the groups using clustering analysis. After that, we perform the PCA by the feeling impression or feature quantity of sound signal each frequency domains.

Furthermore we analyze relationship between feeling impression, and Fluctuation or Residual through the correspondence relationship of principal component axis.

\section{Investigation between 3 Parameters Accompanying on the Calculation Fluctuation value (3PACF) and Feeling Impression}

\subsection{Fluctuation and $3 P A C F$}

Among fluctuations, the well-known 1/f fluctuation means that the power spectrum (PS) of a s ignal is proportional to the $1 / f$ of frequency. Moreover, it is pointed out that there is an effect that a human being feels pleasantness ${ }^{1-6}$.

Fig. 1 is shown the conceptual image of PS. Let $Y(f)$ and $\varepsilon(f)$ be the power of PS and its error, respectively. And we define the PS which is shown in Fig. 1 as Eq. (1).

$$
Y(f)=\frac{k}{f^{a}}+\varepsilon(f)
$$

Taking the logarithm of both sides,

$$
\begin{aligned}
\log Y(f) & =\log \left(\frac{k}{f^{a}}+\varepsilon(f)\right)=\log \left(\frac{k}{f^{a}}(1+\widetilde{\varepsilon}(f))\right) \\
& =\log \left(\frac{k}{f^{a}}\right)+\log (1+\widetilde{\varepsilon}(f)) \\
\log Y(f) & =-a \log f+\log k+\log (1+\widetilde{\varepsilon}(f))
\end{aligned}
$$

Let $y(f)=\log Y(f)$ and $\hat{\varepsilon}(f)=\log (1+\widetilde{\varepsilon}(f))$, respectively. And let $b=\log k$, then we have the following Eq. (3).

$$
\begin{aligned}
y(f) & =-a \log f+\log k+\hat{\varepsilon}(f) \\
& =-a \log f+b+\hat{\varepsilon}(f)
\end{aligned}
$$

Fig. 2 shows the regression line of Eq. (3). In Fig. 2, the vertical axis and the horizontal axis are logarithm of PS and logarithm of frequency $f$, respectively. In this paper, we define the absolute-degree of the regression line $a$ "Fluctuation". Then, we define the intercept of the regression line ("Intercept") as $b$, and we also define the error from the line as $e . .^{8}$

Furthermore, we define the sum of squared errors ("Residual") as Eq. (4). In this equation, $y$ and $Y$ mean the actual measurement value and the theoretical value, respectively.

$$
s=\sum_{i} e_{i}^{2}=\sum_{i}\left(y_{i}-Y_{i}\right)^{2}
$$

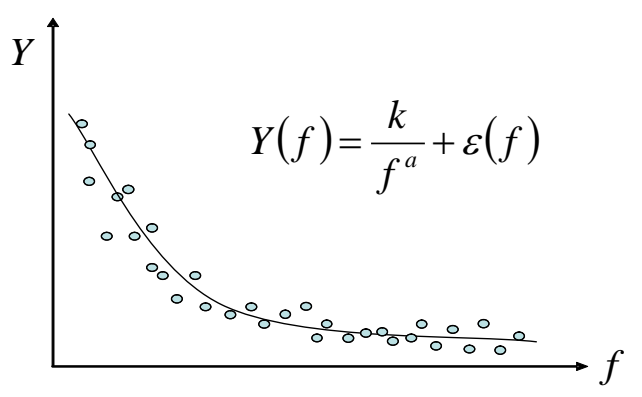

Fig. 1. Conceptual image of PS curve. ${ }^{14,15,17}$ 


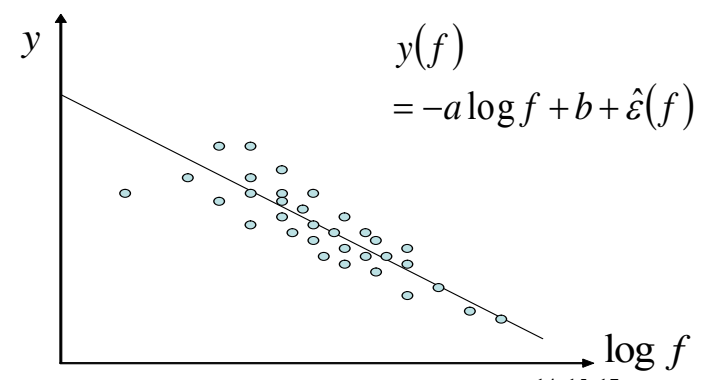

Fig. 2. Example of regression line. ${ }^{14,15,17}$

\subsection{Evaluation of feeling impression}

Next, we have used questionnaire survey in order to investigate the relation between $3 \mathrm{PACF}$ and feeling impression of music. The examinees are 34 students in the age of early twenties. The list of music used in this survey is shown in Table 1.

These sampling frequency and file format are $44.1 \mathrm{kHz}$ and 16 bit wav, respectively. The files are ripped from CD using "Exact Audio Copy V1.0 Beta 3" with Pioneer BDR-S03J BD-drive.

For every piece of the music, we have taken 20 seconds to play it. The examinees evaluated the 4 items as shown in Table 2, by scoring from one to four. Also they have judged the preference for each of music by scoring from one to ten.

Table 1. Music list of wave files. ${ }^{14,17}$

\begin{tabular}{|r|l|c|}
\hline No & \multicolumn{1}{|c|}{ Title (wav) } & Genre \\
\hline \hline 1 & Another Sky & Easy Listening \\
\hline 2 & Londonderry Air & Classic \\
\hline 3 & Blieve you & Easy Listening \\
\hline 4 & Drafting & Easy Listening \\
\hline 5 & Down by the Riverside & Jazz \\
\hline 6 & Space Odessey3 Revelation & Easy Listening \\
\hline 7 & TOMORROW & Pops \\
\hline 8 & Old French Song & Classic \\
\hline 9 & Freedom & Pops \\
\hline 10 & Red River Valley (brass) & Jazz \\
\hline
\end{tabular}

Table 2. Evaluation items of the questionnaire survey. ${ }^{17}$

\begin{tabular}{|l|c|c|c|}
\hline Item1 & Slow & $1 \Leftrightarrow 4$ & Quick \\
\hline Item2 & Heavy & $1 \Leftrightarrow 4$ & Light \\
\hline Item3 & Natural & $1 \Leftrightarrow 4$ & Artificial \\
\hline Item4 & Negative & $1 \Leftrightarrow 4$ & Positive \\
\hline Preference & Dislike & $1 \Leftrightarrow 10$ & Like \\
\hline
\end{tabular}

\subsection{Clustering analysis}

Subsequently, we have conducted clustering analysis to divide into examinees groups who was similar feeling impression, by using the results of questionnaire survey. As preprocessing, we convert the evaluation (Item1 $\sim$ Item4) and Preference as follows;

- $\quad\{$ Item $1 \sim$ Item 4$\} \geq 3 \rightarrow 1$

- $\quad\{$ Item $1 \sim$ Item 4$\} \leq 2 \rightarrow 0$

- Preference $\geq 6 \rightarrow 1$

- Preference $\leq 5 \rightarrow 0$

Therefore, the data of whole examinees are consist from 34 set of 50 dimensional ((Item1 $\sim$ Item4, and Preference) $\times 1$ 0songs) data. That is, this clustering analysis is performed in the 50-dimensional space. And we adopt Ward method ${ }^{9}$ as the analysis.

Ward method is the method which uses often in the clustering analysis. As its characteristics, it is known that it hardly occur the Chain Effect ${ }^{9}$. In this method, the distance of clusters between $C_{i}$ and $C_{j}$ is defined by Eq. (5), and it is fused from small cluster of distance.

In Eq. (5), $d(x, y)$ and $\mu_{i j}$ are Euclidean distance and the mean vector of the cluster that fused the cluster $C_{i}$ and $C_{j}$.

$$
\begin{gathered}
D\left(C_{i}, C_{j}\right)= \\
\sum_{\boldsymbol{x} \in C_{i}, C_{j}} d\left(\boldsymbol{x}, \boldsymbol{\mu}_{\boldsymbol{i} \boldsymbol{j}}\right)^{2}-\left(\sum_{\boldsymbol{x} \in C_{i}} d\left(\boldsymbol{x}, \boldsymbol{\mu}_{\boldsymbol{i}}\right)^{2}+\sum_{\boldsymbol{x} \in C_{j}} d\left(\boldsymbol{x}, \boldsymbol{\mu}_{\boldsymbol{j}}\right)^{2}\right)
\end{gathered}
$$

Fig. 3 shows the concept of cluster fusion process by using Ward method. In the figure, cluster $C_{i}, C_{j}$, and $C_{k}$ have mean vectors $\mu_{i}, \mu_{j}$, and $\mu_{k}$, respectively. Because the distance between $C_{j}$ and $C_{k}$ is the smallest in Fig. 3, they will be fused.

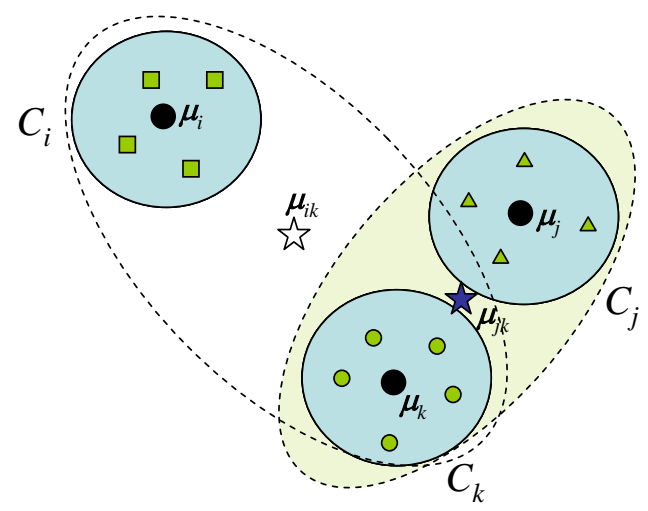

Fig. 3. Conceptual image of cluster fusion process by using Ward method.

And then, we perform Wilk's Lambda test as the statistical test ${ }^{9}$. It is a test of the difference between the 
mean values of the vector comprising a plurality of variables. The null hypothesis $\mathrm{H}_{0}$ and alternative hypothesis $\mathrm{H}_{1}$ are as follows;

$\mathrm{H}_{0}$ : Difference of all mean vectors are equal.

$\mathrm{H}_{1}$ : Difference of all mean vectors are not equal.

Whenever each time of clustering has finished, we apply Wilk's Lambda Test repeatedly. In this test, we set significance to $1 \%$. And then, if it will not come into effect significance of $1 \%$ on next-time test, the clustering is quit ${ }^{10,15,16}$.

\subsection{Principal Component Analysis (PCA)}

In this section, we describe the $\mathrm{PCA}^{12,13}$ performing space and the set of vectors. However, we decide to eliminate Intercept because it substantially equal to the volume of sound.

First, we define Physical Quantity Space (PQS) that is two-dimensional space which axis is the fluctuation value and Residual. The subject of PCA is a set of 10 pieces of music vectors (Table 3 ), and also we perform PCA in each frequency domain (AF, LF, MF, and HF). The other hand, we define Feeling Adjective Space (FAS) that is five-dimensional space which axis is the feeling impression of the examinees. The subject of PCA is a set of 10 pieces of music's Feeling Impression Vectors (FIVs).

Table 3. Fluctuation and Residual. ${ }^{17}$

\begin{tabular}{|c|c|c|c|c|c|c|c|c|c|}
\hline \multirow[b]{2}{*}{ No. } & \multirow{2}{*}{ Title (*.wav) } & \multicolumn{2}{|c|}{$\overline{\mathrm{AF}}$} & \multicolumn{2}{|c|}{$\mathrm{LF}$} & \multicolumn{2}{|c|}{ MF } & \multicolumn{2}{|c|}{$\mathrm{HF}$} \\
\hline & & Fl. & Re. & Fl. & Re. & Fl. & Re. & Fl. & Re. \\
\hline & Another_Sky & 1.715 & 0.414 & 0.454 & 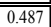 & 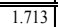 & \begin{tabular}{c|}
0.674 \\
\end{tabular} & (1.777| & 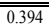 \\
\hline & Londonderry Air & 1.627 & 0.399 & 1.023 & 0.489 & 1.873 & 0.906 & 1.706 & 0.369 \\
\hline & Blieve you & 2.111 & 0.466 & 0.457 & 0.503 & 0.527 & 0.844 & 2.472 & 0.409 \\
\hline & Drafting & 1.593 & 0.431 & 0.917 & 0.474 & 1.296 & 1.006 & 1.706 & 0.404 \\
\hline & Down by the Riverside & 1.724 & 0.448 & 0.553 & 0.502 & 0.470 & 0.679 & 2.115 & 0.406 \\
\hline & Space Odessey3 Revelation & 0.990 & 0.323 & 1.614 & 0.195 & 1.522 & 0.470 & 1.004 & 0.316 \\
\hline & Tomorrow & 2.268 & 0.555 & 0.129 & 0.619 & 0.721 & 0.545 & 2.903 & 0.463 \\
\hline & Old French Song & 1.398 & 0.433 & 1.509 & 0.275 & 1.633 & 1.255 & 1.515 & 0.399 \\
\hline & Freedom & 2.173 & 0.589 & -0.259 & 0.535 & $\frac{-0.047}{-0.047}$ & 0.575 & 2.705 & 0.517 \\
\hline & Red River Valley (brass) & 1.520 & 0.526 & 0.499 & 0.653 & 0.723 & 0.733 & 1.658 & 0.507 \\
\hline
\end{tabular}

Fl.: Fluctuation, Re.: Residual

\subsubsection{Feeling Impression Vector (FIV)}

This subsection, we describe the FIVs.

First, we define the evaluation $\boldsymbol{S}_{(i, k)}$ of examinee $k$ as Eq. (6). $i$ and $e_{1}, \ldots, e_{5}$ are the music number and the evaluation of Item $1 \sim$ Preference, respectively.

$$
\boldsymbol{S}_{(i, k)}=\left(e_{1}, \ldots, e_{5}\right)
$$

Then, we convert the evaluation (Item $1 \sim$ Item 4$)$ and
Preference as same as Section 2.2. And we define the converting processed vector, as Eq. (7). $a_{1}, \ldots, a_{5}$ are zero or one in Eq. (7). That is;

- $\{$ Item $1 \sim$ Item 4$\} \geq 3 \rightarrow 1$

- $\quad\{$ Item $1 \sim$ Item 4$\} \leq 2 \rightarrow 0$

- Preference $\geq 6 \rightarrow 1$

- Preference $\leq 5 \rightarrow 0$

$$
\widetilde{\boldsymbol{S}}_{(i, k)}=\left(a_{1}, \cdots, a_{5}\right)
$$

Hence, the vector $S_{i}{ }^{*}$ of the sum of evaluation $r$ people is FIV which is defined by Eq. (8). $Z_{1}, \ldots, Z_{5}$ are the sum of evaluation Item $1 \sim$ Preference of $r$ people, respectively.

$$
\boldsymbol{S}_{i}^{*}=\left(Z_{1}, \ldots, Z_{5}\right)=\sum_{k=1}^{r} \widetilde{\boldsymbol{S}}_{(i, k)}
$$

That is, we apply Eq. (6) Eq. (9) to each group, which is divided by using clustering analysis, and we perform PCA of the each group.

Subsequent sections, we define $\boldsymbol{u}_{n}(n=1,2)$ and $\boldsymbol{v}_{m}(m=1$, $\ldots, 5)$ in the principal component axis of set of vectors on PQS and FAS, respectively.

Additionally, we define $\boldsymbol{f}_{i}$ and $\boldsymbol{x}_{i}$ in the vectors of music number $i(i=1, \ldots, 10)$ on PQS and FAS, respectively. So we can express $\boldsymbol{f}_{i}$ and $\boldsymbol{x}_{i}$ on the coordinate axes $\boldsymbol{u}_{n}$ and $\boldsymbol{v}_{m}$ respectively as follows.

$$
\left(\left\langle\boldsymbol{f}_{i} \mid \boldsymbol{u}_{1}\right\rangle,\left\langle\boldsymbol{f}_{i} \mid \boldsymbol{u}_{2}\right\rangle\right), \quad\left(\left\langle\boldsymbol{x}_{i} \mid \boldsymbol{v}_{1}\right\rangle, \cdots,\left\langle\boldsymbol{x}_{i} \mid \boldsymbol{v}_{5}\right\rangle\right)
$$

\subsection{Correlation of the principal component axis}

Subsequently, we investigate the correspondence of principal component axes of PQS and principal component axes of FAS. Let $\boldsymbol{C}_{\boldsymbol{u}}$ and $\boldsymbol{C}_{\boldsymbol{v}}$ be the coefficient matrix which is calculated by PCA of PQS and the coefficient matrix which is calculated by PCA of FAS, respectively. And we can describe the basis vectors $\boldsymbol{u}_{n}$ and $\boldsymbol{v}_{m}$ by using $\boldsymbol{p}_{n}$ (the basis vectors on PQS) and $\boldsymbol{i}_{m}$ (the basis vectors on FAS), as shown in Eq. (10) and (11).

$\boldsymbol{u}_{n} \equiv \boldsymbol{C}_{u} \boldsymbol{p}_{n}=\left[\begin{array}{ll}C_{u 11} & C_{u 12} \\ C_{u 21} & C_{u 22}\end{array}\right]\left[\begin{array}{l}\delta_{n 1} \\ \delta_{n 2}\end{array}\right], \delta_{n k}=\left\{\begin{array}{l}(n=k) 1 \\ (n \neq k) 0\end{array}\right.$

$\boldsymbol{v}_{m} \equiv \boldsymbol{C}_{\boldsymbol{v}} \boldsymbol{i}_{m}=\left[\begin{array}{ccc}C_{v 11} & \cdots & C_{v 15} \\ \vdots & \ddots & \vdots \\ C_{v 51} & \cdots & C_{v 55}\end{array}\right]\left[\begin{array}{c}\delta_{m 1} \\ \vdots \\ \delta_{m 5}\end{array}\right], \delta_{m k}=\left\{\begin{array}{l}(m=k) 1 \\ (m \neq k) 0\end{array}\right.$ 
From Eq. (9) (11), correspondence between $\boldsymbol{u}_{n}$ and $\boldsymbol{v}_{m}$ can be judged by the correlation coefficient $R$ between $\left\langle\boldsymbol{f}_{i} \mid \boldsymbol{u}_{n}\right\rangle$ and $\left\langle\boldsymbol{x}_{i} \mid \boldsymbol{v}_{m}\right\rangle \cdot R$ is defined by Eq. (12).

$$
R=\frac{\sum\left\langle\boldsymbol{f}_{i} \mid \boldsymbol{u}_{n}\right\rangle\left\langle\boldsymbol{x}_{i} \mid \boldsymbol{v}_{m}\right\rangle}{\sqrt{\sum\left\langle\boldsymbol{f}_{i} \mid \boldsymbol{u}_{n}\right\rangle^{2}} \sqrt{\sum\left\langle\boldsymbol{x}_{i} \mid \boldsymbol{v}_{m}\right\rangle^{2}}}
$$

\section{Results of Feeling Impression and Discussion}

\subsection{Clustering analysis}

Fig. 4 shows the result of clustering analysis and its statistical test. As shown in Fig. 4, 34-person examinees are divided 3 groups. The names of groups (G1, G2, and G3) are given in descending order of head-count. Examinees belonging to $\mathrm{A}, \mathrm{B}$, and $\mathrm{C}$ are 19 person (55.9\% of total), 8 person ( $23.5 \%$ of total), and 7 person (20.6\% of total), respectively. Table 4 a lso shows the results of the aggregate of evaluation items that is preprocessed describing in Section 2.3.

\subsection{PCA}

Table 5 and Fig. 5 show the results of PCA on PQS (Physical Quantity Space). The plotted marks in Fig. 5 indicate endpoints of the vectors from origin. F rom Table 5 and Fig. 5, we consider that the music that is used in the investigation of this paper, the weight of Fluctuation and the Residual are same degree. Because the components of the Fluctuation and Residual are contained in $\boldsymbol{u}_{1}$ and $\boldsymbol{u}_{2}$ approximately equal proportions. The other hand, Table 6 and Fig. 6 show the results of PCA on FAS (Feeling Adjective Space). In the space, we do not consider principal component $\boldsymbol{v}_{3}$, because the cumulative contribution ratio of principal component $\boldsymbol{v}_{1}$ and $\boldsymbol{v}_{2}$ are about $94.7 \%$ in minimum of each groups. From Table 6 and Fig. 6, we understand as follows;

- In group G1, $\boldsymbol{v}_{2}$ is Preference, and the correspondence between $\boldsymbol{v}_{1}$ and Item2 (Lightness) or Item3 (Artificial) are also strong.

- In group G2 and G3, the correspondence between $\boldsymbol{v}_{1}$ and Item $4, \boldsymbol{v}_{2}$ and Item 1 are strong respectively.

That is, $56 \%$ of examinees (G1) prefer light and artificial music in which have used this paper. However tendency of music to suit the taste of the remaining of examinees (G2 and G3) are not clear, because there are individual differences. From Fig. 6(a), we consider that examinees of group G1 prefer "Believe_you".

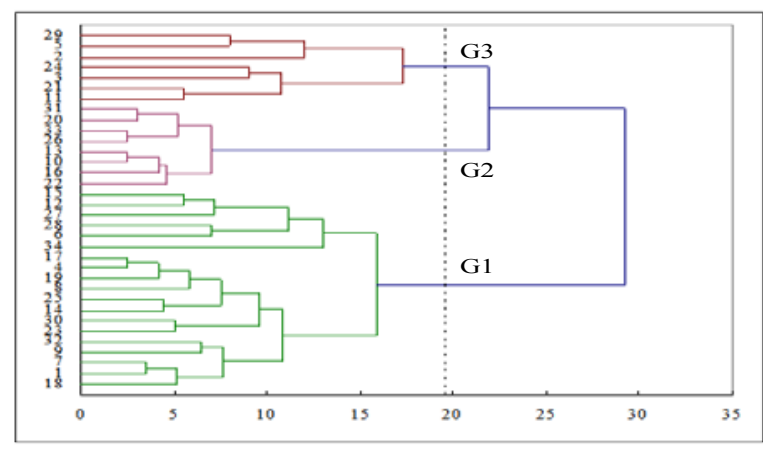

Fig. 4. Dendrogram of clustering result by using questionnaire results of examinees. ${ }^{17}$

Table 4. Results of feeling impression questionnaire. ${ }^{17}$

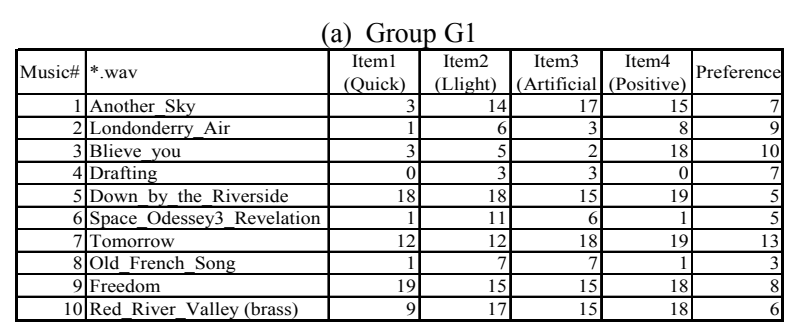

(b) Group G2

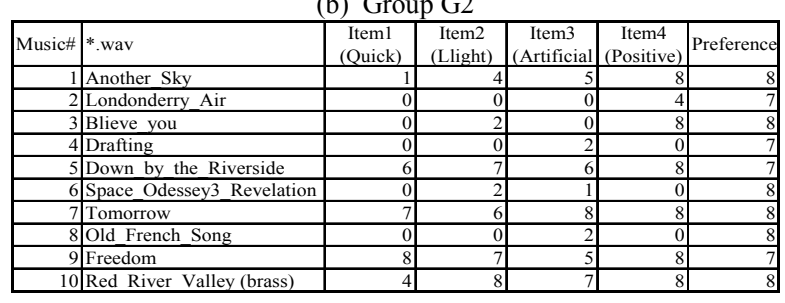

(c) Group G3

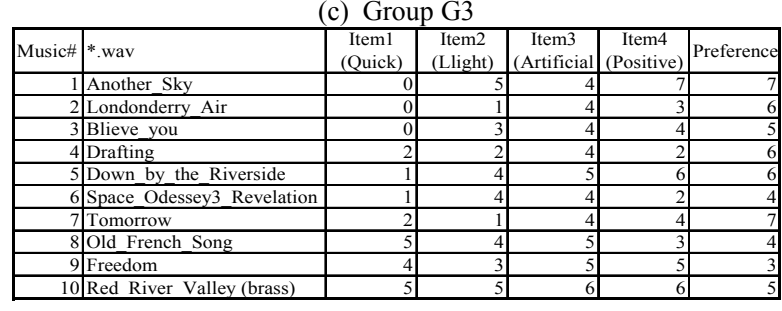

\subsection{Correlation of the principal component axis}

We have calculated the correlation coefficient between principal component axes of PQS $\left(\boldsymbol{u}_{n}\right)$ and FAS $\left(\boldsymbol{v}_{m}\right)$ based on the results of PCA on each space, in each frequency domains (AF, LF, MF, and $\mathrm{HF}$ ).

Table 7 and Fig. 7 show the results of correlation 


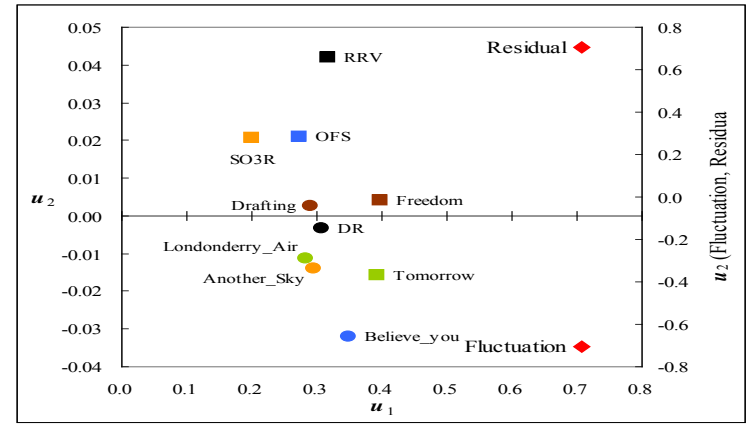

(a) AF (All Frequency domain)

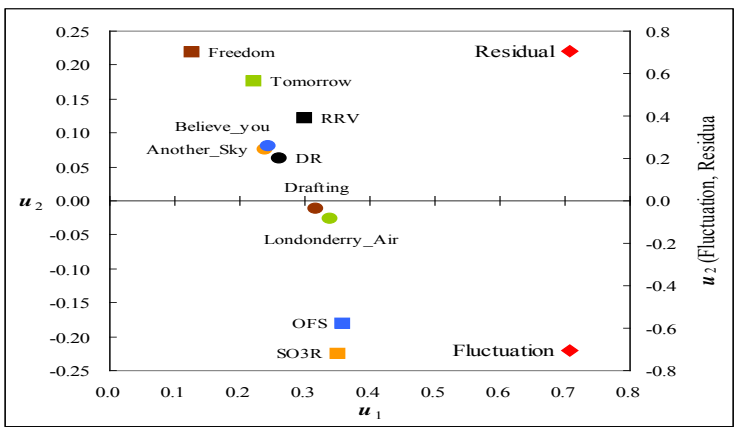

(b) LF (Low Frequency domain)

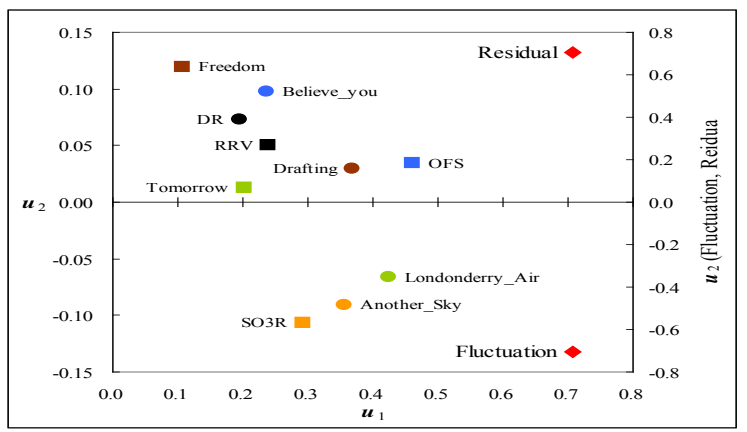

(c) MF (Middle Frequency domain)

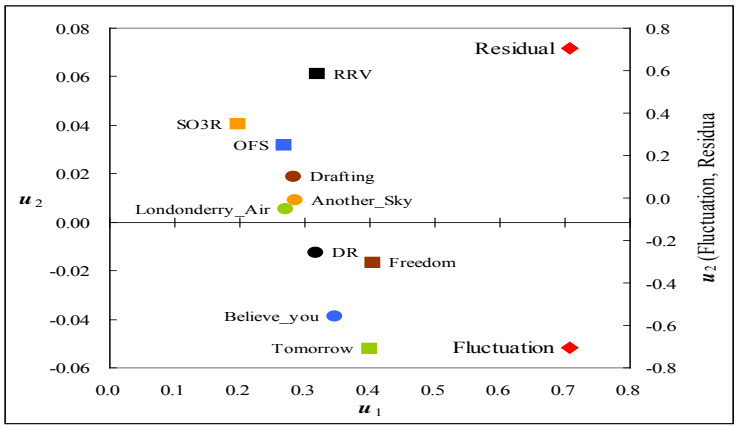

(d) HF (High Frequency domain)

Fig. 5. PCA results on Physical Quantity Space (PQS). ${ }^{17}$ DR: Down_by_the_Riverside, SO3R: Space_Odessey3_Reveration, OFS: Old_French_Song, RRV: Red_River_Valley (brass)

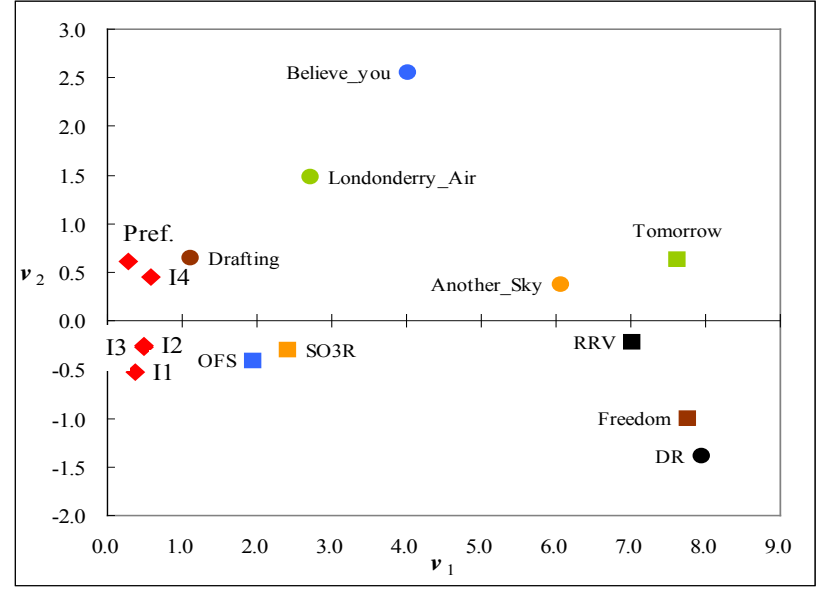

(a) Group G1

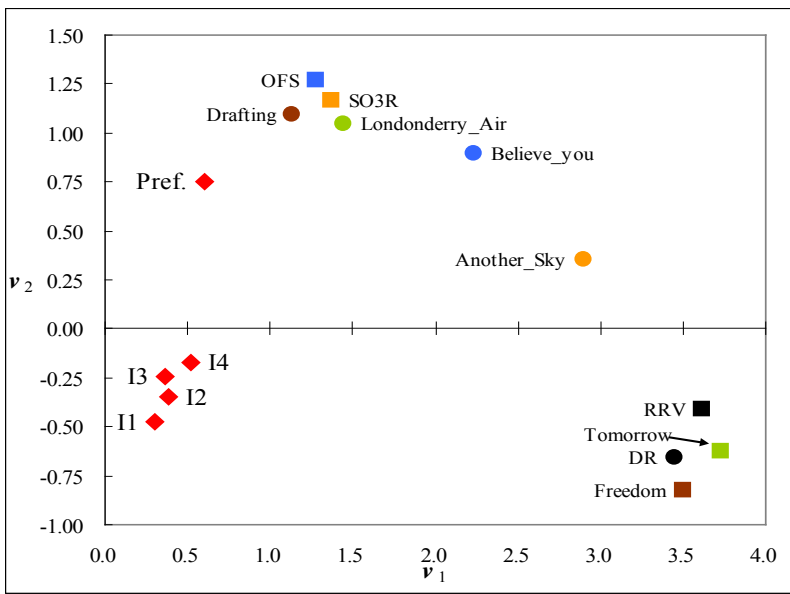

(b) Group G2

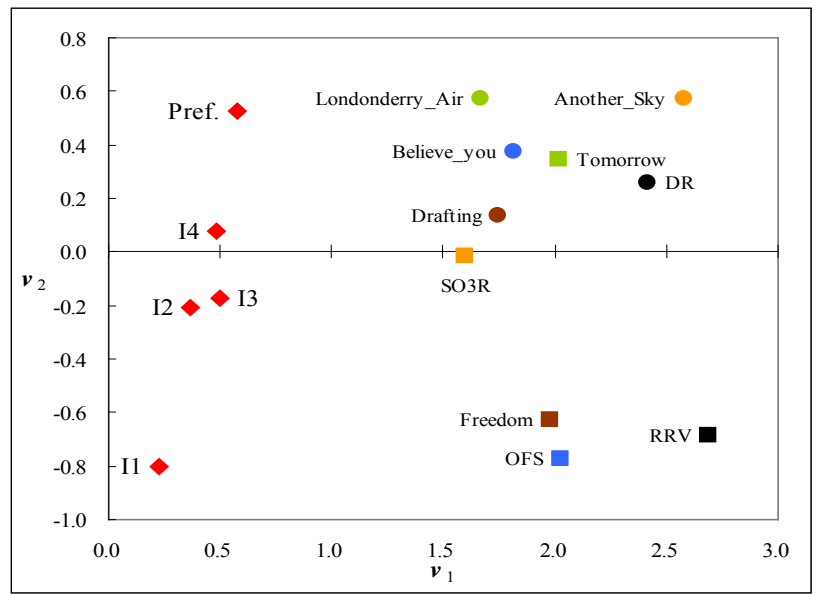

(c) Group G3

Fig. 6. PCA results on Feeling Adjective Space (FAS). ${ }^{17}$ DR: Down_by_the_Riverside, SO3R: Space_Odessey3_Reveration, OFS: Old_French_Song, RRV: Red_River_Valley (brass),

I1: Item1 (Quick), I2: Item2 (Light), I3: Item3 (Artificial), I4: Item4 (Positive), Pref.: Preference (Like) 
Table 5. PCA results on Physical Quantity Space (PQS).

\begin{tabular}{|c|c|c|c|c|c|c|c|c|c|}
\hline \multirow[b]{2}{*}{ No. } & \multirow{2}{*}{ Title (*.wav) } & \multicolumn{2}{|c|}{$\overline{\mathrm{AF}}$} & \multicolumn{2}{|c|}{$\overline{\mathrm{LF}}$} & \multicolumn{2}{|c|}{$\overline{\mathrm{MF}}$} & \multicolumn{2}{|c|}{$\overline{\mathrm{HF}}$} \\
\hline & & $\left\langle f_{i} \mid u_{1}\right\rangle$ & $\left\langle f_{i} \mid u_{2}\right\rangle$ & $\left\langle f_{i} \mid \boldsymbol{u}_{1}\right\rangle$ & $\left\langle f_{i} \mid \boldsymbol{u}_{2}\right\rangle$ & $\left\langle f_{i} \mid u_{1}\right\rangle$ & $\left\langle f_{i} \mid \boldsymbol{u}_{2}\right\rangle$ & $\left\langle f_{i} \mid \boldsymbol{u}_{1}\right\rangle$ & $\left\langle f_{i} \mid \boldsymbol{u}_{2}\right\rangle$ \\
\hline & Another Sky & 0.2959 & -0.0139 & 0.2379 & 0.0757 & 0.3567 & -0.0907 & 0.2856 & 0.0093 \\
\hline & Londonderry Air & 0.2826 & -0.0112 & 0.3402 & $\begin{array}{l}-0.0256 \\
\quad-1\end{array}$ & 0.4235 & \begin{tabular}{|c|c|}
-0.0659 \\
\end{tabular} & 0.2707 & 0.0054 \\
\hline & Believe you & 0.3492 & -0.0319 & 0.2437 & 0.0802 & 0.2354 & 0.0978 & 0.3454 & -0.0390 \\
\hline & Drafting & 0.2904 & 0.0027 & 0.3163 & -0.0115 & 0.3679 & 0.0292 & 0.2838 & 0.0186 \\
\hline & Down by the Riverside & 0.3082 & -0.0032 & 0.2604 & 0.0625 & 0.1955 & 0.0726 & 0.3162 & -0.0127 \\
\hline & Space Odessey3 Revelation & 0.1994 & 0.0206 & 0.3514 & -0.2257 & 0.2915 & -0.1062 & 0.1965 & 0.0404 \\
\hline & Tomorrow & 0.3937 & -0.0158 & 0.2220 & 0.1759 & 0.2017 & 0.0132 & 0.3991 & -0.0523 \\
\hline & Old French Song & 0.2736 & 0.0211 & 0.3582 & -0.1814 & 0.4610 & 0.0343 & 0.2671 & 0.0315 \\
\hline & Freedom & 0.3966 & 0.0042 & 0.1259 & 0.2185 & 0.1072 & 0.1195 & 0.4039 & -0.0168 \\
\hline & Red River Valley (brass) & 0.3164 & 0.0419 & 0.2992 & 0.1210 & 0.2390 & 0.0502 & 0.3187 & 0.0609 \\
\hline
\end{tabular}

(b) Bases vector

\begin{tabular}{|l|c|c|c|c|c|c|c|c|}
\hline Physical & \multicolumn{2}{|c|}{$\mathrm{AF}$} & \multicolumn{2}{c|}{ LF } & \multicolumn{2}{c|}{ MF } & \multicolumn{2}{c|}{ HF } \\
\cline { 2 - 9 } parameter & $\boldsymbol{u}_{1}$ & $\boldsymbol{u}_{2}$ & $\boldsymbol{u}_{1}$ & $\boldsymbol{u}_{2}$ & $\boldsymbol{u}_{1}$ & $\boldsymbol{u}_{2}$ & $\boldsymbol{u}_{1}$ & $\boldsymbol{u}_{2}$ \\
\hline Fluctuation & 0.7071 & -0.7071 & 0.7071 & -0.7071 & 0.7071 & -0.7071 & 0.7071 & -0.7071 \\
\hline Residual & 0.7071 & 0.7071 & 0.7071 & 0.7071 & 0.7071 & 0.7071 & 0.7071 & 0.7071 \\
\hline
\end{tabular}

Table 6. PCA results on Feeling Adjective Space (FAS).

(a) Music vector

\begin{tabular}{|c|c|c|c|c|c|c|c|}
\hline \multirow{2}{*}{ No. } & \multirow{2}{*}{ Title (*.wav) } & \multicolumn{2}{|c|}{ G1 } & \multicolumn{2}{|c|}{$\mathrm{G} 2$} & \multicolumn{2}{|c|}{ G3 } \\
\hline & & $\left\langle\boldsymbol{x}_{i} \mid \boldsymbol{v}_{1}\right\rangle$ & $<\boldsymbol{x}_{i} \mid \boldsymbol{v}_{2}>$ & $\left\langle\boldsymbol{x}_{i} \mid \boldsymbol{v}_{1}\right\rangle$ & $\left\langle\boldsymbol{x}_{i}\right| \boldsymbol{v}_{2}>$ & $\left\langle\boldsymbol{x}_{i} \mid \boldsymbol{v}_{1}\right\rangle$ & $<\boldsymbol{x}_{i} \mid \boldsymbol{v}_{2}>$ \\
\hline & Another Sky & 6.0797 & 0.3689 & 2.8965 & 0.3500 & 2.5807 & 0.5734 \\
\hline & Londonderry Air & 2.7105 & 1.4754 & 1.4441 & 1.0455 & 1.6698 & 0.5723 \\
\hline 3 & Believe you & 4.0316 & 2.5531 & 2.2330 & 0.8974 & 1.8146 & 0.3734 \\
\hline 4 & Drafting & 1.1179 & 0.6379 & 1.1369 & 1.0948 & 1.7465 & 0.1387 \\
\hline & Down by the Riverside & 7.9547 & $\begin{array}{ll}-1.3869 \\
\end{array}$ & 3.4447 & -0.6613 & 2.4187 & 0.2587 \\
\hline & Space Odessey3 Revelation & 2.4144 & -0.3028 & 1.3663 & 1.1635 & 1.5952 & -0.0153 \\
\hline & Tomorrow & 7.6395 & 0.6232 & 3.7316 & -0.6296 & 2.0180 & 0.3437 \\
\hline & Old French Song & 1.9509 & -0.4201 & 1.2753 & 1.2671 & 2.0279 & -0.7724 \\
\hline 9 & Freedom & 7.7726 & $\begin{array}{ll}-1.0138 \\
\end{array}$ & 3.4965 & -0.8230 & 1.9809 & -0.6257 \\
\hline 10 & Red River Valley (brass) & 7.0231 & -0.2201 & 3.6188 & -0.4069 & 2.6909 & -0.6853 \\
\hline
\end{tabular}

(b) Bases vector

\begin{tabular}{|l|r|r|r|r|r|r|}
\hline \multicolumn{1}{|c|}{$\begin{array}{c}\text { Feeling } \\
\text { impression }\end{array}$} & \multicolumn{2}{|c|}{$\mathrm{G} 1$} & \multicolumn{2}{|c|}{$\mathrm{G} 2$} & \multicolumn{2}{c|}{$\mathrm{G} 3$} \\
\cline { 2 - 7 } & $\boldsymbol{v}_{1}$ & $\boldsymbol{v}_{2}$ & $\boldsymbol{v}_{1}$ & $\boldsymbol{v}_{2}$ & $\boldsymbol{v}_{1}$ & \multicolumn{1}{c|}{$\boldsymbol{v}_{2}$} \\
\hline Item1 (Quick) & 0.366 & -0.520 & 0.296 & -0.474 & 0.227 & -0.801 \\
\hline Item2 (Light) & 0.482 & -0.249 & 0.382 & -0.348 & 0.364 & -0.209 \\
\hline Item3 (Artificial) & 0.477 & -0.269 & 0.367 & -0.243 & 0.495 & -0.174 \\
\hline Item4 (Positive) & 0.570 & 0.460 & 0.518 & -0.175 & 0.483 & 0.078 \\
\hline Preference (Like) & 0.285 & 0.619 & 0.603 & 0.751 & 0.581 & 0.527 \\
\hline
\end{tabular}

Table 7. Correlation coefficient between principal. ${ }^{17}$

(a) Group G1

\begin{tabular}{|c|c|c|c|c|c|c|c|c|}
\hline $\begin{array}{c}\text { Principal } \\
\text { axis }\end{array}$ & $\boldsymbol{u}_{1}$ & $\boldsymbol{u}_{2}$ & $\boldsymbol{u}_{1}$ & $\boldsymbol{u}_{2}$ & $\boldsymbol{u}_{1}$ & $\boldsymbol{u}_{2}$ & $\boldsymbol{u}_{1}$ & $\boldsymbol{u}_{2}$ \\
\hline $\boldsymbol{v}_{1}$ & 0.926 & 0.028 & 0.774 & 0.547 & 0.704 & 0.351 & 0.931 & -0.059 \\
\hline $\boldsymbol{v}_{2}$ & 0.216 & -0.579 & 0.230 & 0.068 & 0.288 & -0.080 & 0.203 & -0.294 \\
\hline
\end{tabular}

(b) Group G2

\begin{tabular}{|c|c|c|c|c|c|c|c|c|}
\hline $\begin{array}{c}\text { (b) Group } \mathrm{G} 2 \\
\text { Principal } \\
\text { axis }\end{array}$ & $\boldsymbol{u}_{1}$ & $\boldsymbol{u}_{2}$ & $\boldsymbol{u}_{1}$ & $\boldsymbol{u}_{2}$ & $\boldsymbol{u}_{1}$ & $\boldsymbol{u}_{2}$ & $\boldsymbol{u}_{1}$ & $\boldsymbol{u}_{2}$ \\
\hline $\boldsymbol{v}_{1}$ & 0.956 & 0.049 & 0.827 & 0.506 & 0.761 & 0.341 & 0.960 & -0.015 \\
\hline $\boldsymbol{v}_{2}$ & 0.251 & 0.000 & 0.528 & -0.660 & 0.611 & -0.351 & 0.233 & 0.371 \\
\hline
\end{tabular}

(c) Group G3

\begin{tabular}{|c|c|c|c|c|c|c|c|c|}
\hline $\begin{array}{c}\text { Principal } \\
\text { axis }\end{array}$ & $\boldsymbol{u}_{1}$ & $\boldsymbol{u}_{2}$ & $\boldsymbol{u}_{1}$ & $\boldsymbol{u}_{2}$ & $\boldsymbol{u}_{1}$ & $\boldsymbol{u}_{2}$ & $\boldsymbol{u}_{1}$ & $\boldsymbol{u}_{2}$ \\
\hline $\boldsymbol{v}_{1}$ & 0.976 & 0.115 & 0.945 & 0.280 & 0.911 & 0.226 & 0.975 & 0.166 \\
\hline $\boldsymbol{v}_{2}$ & 0.024 & -0.784 & 0.031 & 0.083 & 0.075 & -0.425 & 0.016 & -0.482 \\
\hline
\end{tabular}

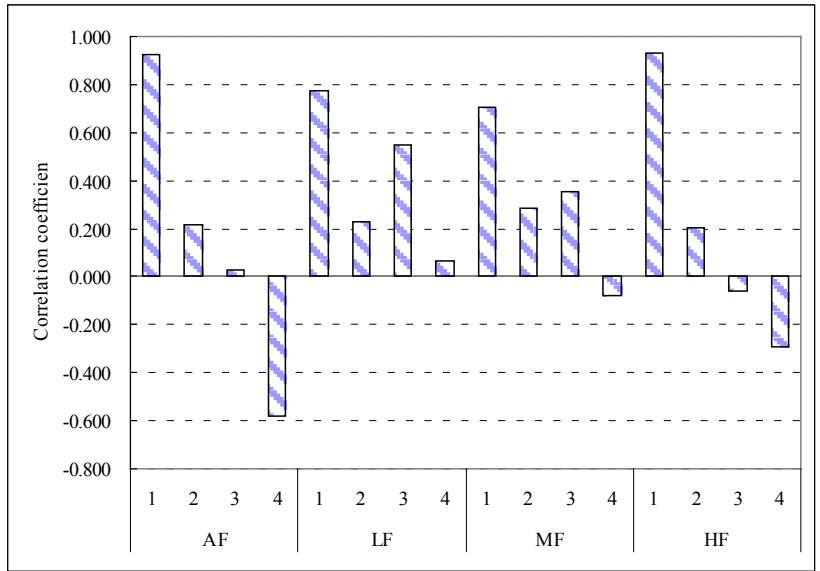

(a) Group G1

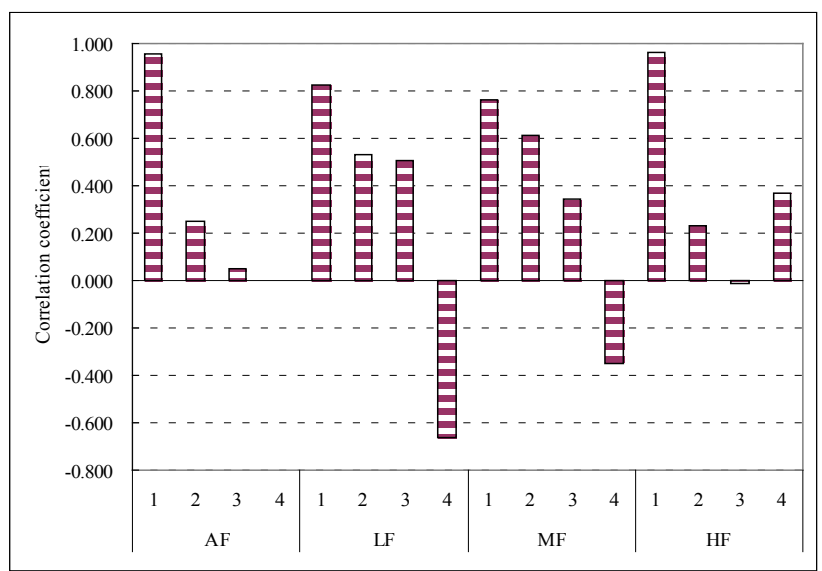

(b) Group G2

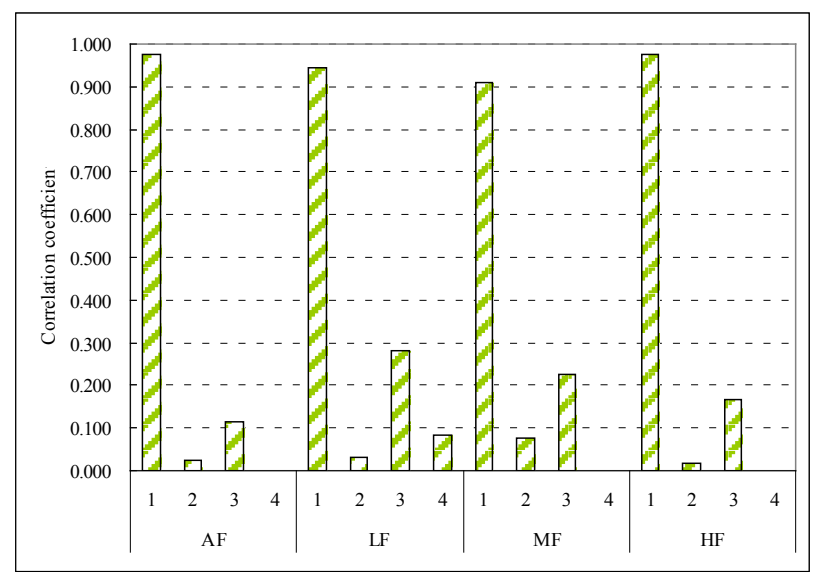

(c) Group G3

Fig. 7. Correlation coefficient between principal axes.

1: Correlation between $\left\langle\boldsymbol{f}_{i}\right| \boldsymbol{u}_{1}>$ and $\left\langle\boldsymbol{x}_{i} \mid \boldsymbol{v}_{1}\right\rangle$,

2: Correlation between $\left\langle\boldsymbol{f}_{i}\right| \boldsymbol{u}_{1}>$ and $<\boldsymbol{x}_{i} \mid \boldsymbol{v}_{2}>$,

3: Correlation between $\left\langle\boldsymbol{f}_{i}\right| \boldsymbol{u}_{2}>$ and $<\boldsymbol{x}_{i}\left|\boldsymbol{v}_{1}\right\rangle$,

4: Correlation between $\left\langle\boldsymbol{f}_{i} \mid \boldsymbol{u}_{2}\right\rangle$ and $\left\langle\boldsymbol{x}_{i} \mid \boldsymbol{v}_{2}\right\rangle$. 
coefficient in each group (G1, G2, and G3). The colored portion of Table 7 indicates that the absolute value of the correlation coefficient is 0.700 or higher.

\subsubsection{Group G1}

From Table 7 (a) and Fig. 7 (a), the correlation coefficient on AF and HF between $\boldsymbol{u}_{1}$ and $\boldsymbol{v}_{1}$ are 0.926 and 0.931 respectively, so they have strong positive correlation.

We refer Fig. 5(a) and (d), increase in Fluctuation and Residual are tendency in response to increase of $\boldsymbol{u}_{1}$. Also from Fig. 6(a), increase in Item2 (Lightness) and Item3 (Artificial) are tendency in response to increase of $v_{1}$.

Similarly, the correlation coefficient on LF and MF between $\boldsymbol{u}_{1}$ and $\boldsymbol{v}_{1}$ are 0.774 and 0.704 respectively, so they have positive correlation.

We refer Fig. 5(a) (c), Fluctuation and Residual on LF of all music are lower than AF. And the music which Fluctuation and Residual are small on AF, there are tendency they increase on MF.

Therefore, we consider the music become light and artificial impression by increasing Fluctuation and Residual, on $\mathrm{AF}, \mathrm{MF}$, and $\mathrm{HF}$.

\subsubsection{Group $G 2$}

From Table 7 (b) and Fig. 7 (b), we understand that G2 have tendency same as G1. And, the correlation coefficient of each domain between $\boldsymbol{u}_{1}$ and $\boldsymbol{v}_{1}$ are higher than 0.761 , so they have positive correlation. Besides, we refer Fig. 6(b), increase in Item4 (Positive) is tendency in response to increase of $\boldsymbol{v}_{1}$.

Therefore, we consider the music become positive impression by increasing Fluctuation and Residual.

\subsubsection{Group G3}

From Table 7 (c) and Fig. 7 (c), we understand that the correlation coefficient of each domain of G3 between $\boldsymbol{u}_{1}$ and $\boldsymbol{v}_{1}$ are higher than 0.911 , so they have strong positive correlation. Especially the correlation coefficient of AF between $\boldsymbol{u}_{2}$ and $\boldsymbol{v}_{2}$ is -0.784 , so they have negative correlation.

We refer Fig. 6(c), increase in Item4 (Positive) is tendency in response to increase of $\boldsymbol{v}_{1}$. And from Fig 5 (a) $\sim$ (d), increase in Fluctuation and Residual are tendency in response to increase of $\boldsymbol{u}_{1}$. Furthermore, decreasing Fluctuation and increasing Residual are tendency in response to decrease of $\boldsymbol{v}_{2}$.

Therefore, we consider the music become positive impression by increasing Fluctuation and Residual. Especially, by increasing Fluctuation and Residual on $\mathrm{AF}$, the music become fast impression.

\subsubsection{Overall tendency}

We understand that $56 \%$ of examinees (Group G1) feel light and artificial impression from the music which both of Fluctuation and Residual are high, and they have tendency that they prefer the music which Fluctuation and Residual are high and low, respectively. We also understand that rest of $44 \%$ examinees (Group G2 and G3) feel positive impression, but they don't.

We can judge that the sensitivity of the music impression is strong influence by Fluctuation and Residual of HF, because the above tendencies are common to $\mathrm{AF}$ and $\mathrm{HF}$.

\section{Conclusion}

In this paper, we have investigated the effects between feature quantity of sound signal and feeling impression by using Principal Component Analysis (PCA). As feature quantity, we have used Fluctuation and Residual. As for the feeling impression questionnaire, we have presented 10 piece of music to examinees and they evaluated 5 items, i.e. quickness, lightness, artificial, positiveness, and preference (like or dislike). Then, we performed clustering analysis using Ward method based on the evaluation results, and we understood that the examinees feeling impression could be divided into 3 groups.

Next, we have performed PCA in the Physical Quantity Spaces of the each frequency domain (AF, LF, MF, and HF) and performed PCA in the Feeling Adjective Spaces of each group. Furthermore, we also investigated correlation between the principal component axes.

As the results, we have understood that $56 \%$ of examinees feel light and artificial impression from the music in which both values of Fluctuation and Residual are high. And we have also found that they prefer such music.

Although there were not seen such correlativity about the rest $44 \%$ of examinees, we were able to understand that they feel positive impression from the music in which the both values of Fluctuation and Residual are high. 
Moreover, we have found that the high frequency feature quantity of sound has the strongest influence to people's feeling impression.

\section{References}

1. T. Musha, Reason why person feels pleasantness and unpleasantness --sound, image, and sense of touch-- that approaches to the mystery of "pleasantness" (Kawade Shobo Shinsya (KAWADE dream new book), 1999) (in Japanese).

2. T. Musha, The world of fluctuation -Mystery of $1 / f$ fluctuations of the world- (Kodansha Bluebacks. Kodansha, 1980) (in Japanese).

3. H. Akaike and T. Wada, Fluctuation and rhythm of living body (Kodansha Scientific, 1997) (in Japanese).

4. S. Horiuchi, Mystery of fluctuation (Shinano Mainichi Newspapers Co. 1997) (in Japanese).

5. E. Teramoto, R. Hirota, T. Musha, and M. Yamaguchi, Infinity, chaos, and fluctuation (Bifukan, 1985) (in Japanese).

6. T. Musha, Conception of fluctuation that approaches to the mystery of 1/f fluctuation (NHK Books, 1998) (in Japanese).

7. T. Musha, Science of fluctuation, Vol.1 10 (Morikita Publication, 1991-1999) (in Japanese).

8. Y. Kawakami, T. Hattori, T. Yamamatsu, T. Izumi, and H. Kawano, Experimental Investigation of the Relation between Feeling Impression and Quantities Accompanying Fluctuation Calculation in Sound Signal, Transactions of Japan Society of Kansei Engineering 10(3), ISSN: 1884-5258 (2011) 365-374 (in Japanese).

9. B. S. Everitt, The Cambridge Dictionary of Statistics, 2nd edn. (Cambridge University Press, 2002).

10. Y. Kawakami, T. Hattori, H. Kawano, T. Izumi, Statistical Investigation on Correlation between Feeling Impression and Feature Quantity of Sound Signal, in Proc. First International Symposium on Affective Engineering 2013, ISSN: 2187-669X (Kita-Kyushu, Fukuoka, 2013), pp.297-302.

11. T. Kado, Basic knowledge of descriptive audio (Ohmsha, 2001) (in Japanese).

12. S. Muto, Statistical Analysis Handbook (Asakura Shoten, 1995) (in Japanese).

13. H. Yanai, Multivariate data analysis - Theory and Applications (Asakura Shoten, 1994) (in Japanese).

14. Y. Kawakami, T. Hattori, T. Yamamatsu, T. Izumi, and H. Kawano, Investigation of Feeling Effect by Feature Quantity Including Fluctuation in Sound Signal, in Proc. The Institute of Electrical Engineers of Japan C (CD) (Kitami, Hokkaido, 2013), pp. 1490-1495 (in Japanese).

15. Y. Kawakami, T. Hatori, H. Kawano, and T. Izumi, Statistical Investigation on Relation between Feeling Impression and Feature Parameters of Sound Signal, International Journal of Affective Engineering 13(1), ISSN: 2187-5413 (2014) 71-80.
16. Y. Kawakami, Research on Correlation between Feature Quantity of Sound Signal and Kansei Impression, Doctoral dissertation, Kagawa University, Graduate School of Engineering (2014) (in printing, in Japanese).

17. Y. Kawakami, T. Hattori, H. Kawano, and T. Izumi, Investigation of Feature Quantity in Sound Signal and Feeling Impression Using PCA, in Proc. International Conference on Artificial Life and Robotics 2015, ISBN 978-4-9902880-9-9 (Oita, Japan, 2015), pp.265-271. 